\title{
DESARROLLO DE COMPETENCIAS EN FÍSICA DESDE EL MODELO DE APRENDIZAJE INVERTIDO
}

\section{DEVELOPMENT OF COMPETENCES IN PHYSICS FROM THE INVERTED LEARNING MODEL}

\section{Cesar Augusto Hernández Suárez ${ }^{1}$}

\section{Audin Aloiso Gamboa Suárez ${ }^{2}$}

Raúl Prada Núñez ${ }^{3}$

\section{RESUMEN}

Con este trabajo se pretende mostrar los beneficios del modelo aula invertida como estrategia para el aprendizaje de la Física en el desarrollo de competencias científicas en estudiantes del nivel de educación media. Se adopta un enfoque cuantitativo de tipo descriptivo. Para ello se seleccionaron contenidos y materiales de apoyo de acuerdo con un proceso de planeación y ejecución de actividades, teniendo en cuenta que invertir el

1 Docente investigador de la Universidad Francisco de Paula Santander. Magíster en Enseñanza de las Ciencias por la Universidad Nacional Experimental del Estado Táchira (Venezuela). Correo electrónico: cesaraugusto@ ufps.edu.co, Orcid: 0000-0002-7974-5560

2 Docente investigador de la Universidad Francisco de Paula Santander. Doctor en Ciencias de la Educación por la Universidad de Cartagena. Correo electrónico: audingamboa@ufps.edu.co, Orcid: 0000-0001-9755-6408

3 Docente investigador de la Universidad Francisco de Paula Santander. Magíster en Ingeniería de Análisis de Datos, Mejora de Procesos y Toma de Decisiones por la Universidad Politécnica de Valencia (España). Correo electrónico: raulprada@ufps.edu.co, Orcid: 0000-0001-6145-1786 aula implica repasar contenidos declarativos de bajo nivel en casa, y que la práctica con alto nivel cognitivo se desarrolle en la clase, con la asesoría del docente. Se diseña un cuestionario en el que se incorporó una escala para determinar la percepción de los estudiantes frente al uso de la estrategia. Los resultados reflejan que más de la mitad de los estudiantes se mostraron satisfechos con la estrategia, lo cual generó una leve mejoría en el rendimiento académico de la asignatura de Física de un periodo académico al otro. Se concluye que los estudiantes prefieren el apoyo de material multimedia frente a otro tipo para el desarrollo de la estrategia aula invertida.

PALABRAS CLAVE: Aula invertida, ciencias naturales, educación básica.

\section{ABSTRACT}

With this work we intend to show the benefits of the invested classroom model as a strategy for 
learning Physics in the development of scientific competences in students of the secondary education level. A quantitative approach of descriptive type is adopted. For this purpose, contents and support materials were selected according to a process of planning and execution of activities, taking into account that investing the classroom implies reviewing low-level declarative contents at home, and that practice with a high cognitive level is developed in class, with the teacher's advice. A questionnaire was designed in which a scale was incorporated to determine the students' perception of the use of the strategy. The results reflect that more than half of the students were satisfied with the strategy, which generated a slight improvement in the academic performance of the Physics subject from one academic period to the next. It is concluded that students prefer multimedia support to other types of support for the development of the inverted classroom strategy.

KEYWORDS: Inverted classroom, natural sciences, basic education.

\section{INTRODUCCIÓN}

Los resultados de aprendizaje de los estudiantes de educación media, especialmente en Ciencias Naturales, no tienen las competencias mínimas para resolver situaciones problémicas en el área. Del resultado del informe PISA 2018, se puedo verificar (centrándose en los dominios de Lectura, Matemáticas y Ciencias) que los estudiantes en Ciencias obtuvieron un rendimiento menor que la media de la OCDE, de los cuales casi el $40,0 \%$ obtuvieron un bajo nivel de logro. Cerca de la mitad de los estudiantes, tiene dificultades para reconocer la explicación correcta de fenómenos científicos familiares y pueden utilizar dicho conocimiento para identificar, en casos sencillos, si una conclusión es válida a partir de los datos proporcionados (Echazarra y Schwabe, 2019). Además, los estudiantes que ingresan a estudiar carreras de ingeniería, en el nivel introductorio presentan dificultades para desarrollar un entendimiento funcional de los conceptos básicos de la Física, dejando como resultado la deserción de la universidad (Flores-García, et al., 2008).

Lo anterior plantea retos a los docentes para encontrar estrategias efectivas para mejorar las habilidades de los estudiantes en Ciencias, y que se deben, entre otras problemáticas, al uso de estrategias tradicionales, que desconocen los diferentes ritmos y estilos de aprendizaje que poseen los estudiantes (Mora-Ramírez \& Hernández-Suárez, 2017). También se necesita motivar al estudiante, para que sea partícipe en la construcción de su aprendizaje. Por otra parte, el uso de las TIC como mediaciones de apoyo al proceso de enseñanza - aprendizaje están transformando la educación de manera favorable (Martín, Hernández-Suarez, \& Mendoza-Lizcano, 2017).

Como respuesta a estas necesidades, el aula invertida, es un modelo innovador que permite al docente utilizar las TIC (de forma asincrónica) para poner a disposición del estudiante (antes de clase), material digital con contenido audiovisual y multimedia, que él estudia a su propio ritmo. Este material debe ser elaborado cumpliendo una serie de requisitos pedagógicos y didácticos que permitan al estudiante mediante aprendizaje autónomo adquirir los conceptos mínimos para abordar las situaciones en clase. Durante el tiempo de la clase, el docente realiza actividades de acompañamiento y retroalimentación a los estudiantes para facilitar la adquisición de conceptos, mediante actividades colaborativas y talleres prácticos, convirtiendo el aula en un ambiente de aprendizaje activo (HernándezSuárez, Prada-Núñez y Gamboa-Suárez, 2020). Todo este proceso es planificado de forma curricular y didáctica por el docente, lo que favorece el desarrollo de competencias y la obtención de mejores resultados de aprendizaje en los estudiantes (Hernández, Prada y Ramírez, 2018). 
Además, el modelo aula invertida, permite al docente una evaluación de los estudiantes acorde a sus características personales, intereses, ritmos de desarrollo y estilos de aprendizaje para valorar sus avances. Esto es un cambio al modelo tradicional del aula, ya que invertir el sentido de la clase se libera tiempo mediante la eliminación de gran parte de la instrucción directa de la clase, para que el maestro pueda supervisar las experiencias de aprendizaje colaborativo, proporcionando la oportunidad para la comunicación entre los diversos estudiantes (Johnson \& Renner, 2012).

Al aprovechar las ventajas que ofrece el aula invertida, se busca hacer un cambio pedagógico mediada por la tecnología, que permita gestionar, dinamizar y optimizar los procesos enseñanza-aprendizaje. De acuerdo con esto el objetivo de este estudio consistió en determinar en qué medida la implementación de un aula invertida, apoya el aprendizaje de la Física en los estudiantes de educación media.

\section{MARCO TEÓRICO Y CONCEPTUAL}

El aula invertida, busca invertir los momentos y roles de la enseñanza tradicional, donde la clase pueda ser dada y consultada por el estudiante mediante herramientas multimedia, de manera que las clases presenciales se conviertan en exploración y práctica de los conocimientos y competencias que se requieren desarrollar. El aula invertida es un modelo mediado por la tecnología que se rota con el aula tradicional.

El término aula invertida es originalmente acuñado por Lage, Platt \& Treglia (2000) como Inverted Classroom (IC). La propuesta de aula invertida incluye el uso de tecnología multimedia (videoconferencias, presentaciones) para acceder al material de apoyo fuera del aula. En 2007, el modelo fue popularizado por Bergmann \& Sams, denominándolo flipped classroom, término más reconocido en el nivel educativo básico.
La propuesta de Bergmann \& Sams (2012), no fue fundamentada en una teoría educativa, si no que durante su implementación se fueron realizando los ajustes sustentados en factores tales como: abarcar los diferentes estilos de aprendizaje del estudiantado, promover un ritmo individual de avance y desarrollar habilidades de aprendizaje autodirigido (Martínez-Olvera, Esquivel-Gámez, y Martínez, 2015a, 2015b).

Los modelos educativos tradicionales, se enfocan a memorizar, retener, conocer $y$ comprender contenidos de manera individual. Estas habilidades cognitivas corresponden a los niveles bajos de la taxonomía propuesta por Bloom (1964). En consecuencia, el espacio en el aula no es aprovechado para actividades que potencien el desarrollo de habilidades más complejas, tales como aplicar, analizar, evaluar, crear, que pueden realizarse en ambientes de aprendizaje fomentando la comunicación entre pares (Hernández-Suárez, Prada y Gamboa, 2020), lo que promueve los ritmos y estilos de aprendizaje, especialmente los del aprendizaje experiencial de Kolb (1984).

Adicionalmente, se fundamenta en la la perspectiva cognitiva de Piaget (1972), especialmente con los principios del constructivismo fundamentado en la resolución de problemas, el aprendizaje activo y el aprendizaje colaborativo; complementado con la perspectiva social de Vygotsky (1978) y su zona de desarrollo próximo de donde se deriva el aprendizaje cooperativo. Lo anterior muestra por un lado la integración entre el proceso cognitivo y como se construye el conocimiento, reconociendo los aprendizajes previos de los estudiantes (Gaviria et al., 2019), y por otro, la relación que se establece entre aprendizaje colaborativo y cooperativo (Durley, 2014).

El aula invertida, implica que las actividades que se realizan en casa son las que se ubican ahora en los niveles más bajos de la taxonomía de Bloom, mientras que aquellas que requieren 
de mayor esfuerzo cognitivo se pueden realizar en el salón con la orientación del profesor (Zainuddin \& Halili, 2016).

Estas características sitúan al aula invertida como un modelo aprendizaje activo, centrado en el estudiante (Prada, Hernández, \& Gamboa, 2019) por lo que está relacionado con el modelo constructivista para facilitar el aprendizaje individualizado y diferenciado (Davies, Dean \& Ball, 2013), centrado en el estudiante, en el cual los estudiantes son los agentes primarios de su propio progreso (Davis, 2013), pero también incluyen la colaboración con los compañeros y la participación en el aprendizaje activo, y tareas interactivas de resolución de problemas.

El modelo de aula invertida se considera como un modelo de aprendizaje híbrido o combinado. En muchas escuelas, este aprendizaje está surgiendo como una innovación al aula tradicional. Este modelo híbrido busca el intento de combinar lo mejor de ambos mundos, esto es, las ventajas del aprendizaje en línea (o apoyado con recursos digitales) combinado con todos los beneficios del aula tradicional (Christensen, Horn \& Staker, 2013).

Finalmente, el modelo de Aula Invertida es aquel en el que la rotación se produce entre la escuela para la práctica (o los proyectos) orientada por el profesor (cara a cara) y en la casa u otra ubicación para el contenido y la instrucción en línea. Por todo lo anterior, este modelo se nutre de métodos y estrategias como el aprendizaje individualizado y diferenciado, el trabajo en equipo (cooperativo - colaborativo), la resolución de problemas y el aprendizaje basado en problemas y proyectos.

\section{MÉTODO}

La investigación que da origen a este producto, adopta un enfoque cuantitativo, con recolección de datos antes, durante y después de la aplicación del aula invertida de aprendizaje. Con estos datos se realiza un procesamiento de ellos a nivel descriptivo, con el objetivo de medir de forma independiente las variables con que se tiene relación.

\section{Las etapas del desarrollo del proyecto.}

Para adoptar este método se deben agotar las siguientes etapas o fases con el fin de garantizar su correcta implementación.

- Diseño del aula invertida de aprendizaje para lo cual se debe realizar la selección de contenidos y actividades.

- Sensibilización de estudiantes que van a hacer parte de la población estudio sobre la importancia de la asignatura, la temática y la plataforma.

- Ejecución de actividades en el aula invertida por lecciones programadas, para lo cual cada lección contará con documentos de apoyo, foros temáticos y chat para resolver dudas, entre muchos otros recursos.

- Durante la clase presencial se ejecuta la aplicación de instrumentos con el fin de validar las actividades de preparación para la clase. Se recomienda la realización de laboratorios con materiales económicos y de fácil adquisición, para explicar la aplicación de la Física (circuitos eléctricos, funcionamiento de los fluidos, movimientos armónico simple, leyes de Newton, entre otros).

- La aplicación de los cuestionarios de evaluación una vez terminadas las lecciones para valorar el aprendizaje de esta.

- Aplicación del pos-test, para comparar el nivel de desempeño del aula en el desarrollo de competencias. 
La población objeto de estudio son estudiantes de educación media de una institución educativa publica del departamento de Norte de SantanderColombia. Se seleccionó como muestra la totalidad de la población 100 estudiantes por ello es un estudio censal, ello con el fin de poder validar específicamente cada uno de los casos teniendo en cuenta el rendimiento académico y el trabajo en el aula invertida.

\section{INSTRUMENTOS}

Test para valorar el aula Invertida de Aprendizaje. Para evaluar la efectividad del aula invertida se diseñó un cuestionario tipo test que consta de 21 ítems, separados por gestiones (administrativa, pedagógica), recurriendo a una escala tipo Likert con cinco niveles de respuesta: Totalmente de acuerdo (5), De acuerdo (4), Ni de acuerdo, ni en desacuerdo (3), En desacuerdo
(2) y Totalmente en desacuerdo (1). Este se aplicó al finalizar la ejecución del proyecto, buscando evaluar la gestión de esta frente a la temática de la asignatura de Física. Para aplicar la prueba final, se tomó una muestra aleatoria simple.

\section{RESULTADOS}

El software utilizado para procesar datos es Microsoft Excel donde primero se genera una matriz general de la totalidad de las respuestas por cada estudiante y posteriormente se van agrupando ítems, de acuerdo con la gestión indicada; por último, se grafican los ítems agrupados para dar descripción a cada uno. Se valida la implementación del Aula Invertida, la cual se midió la gestión administrativa, gestión pedagógica, materiales, estilos y habilidades, interacción en clases sincrónicas y asincrónicas.

Tabla 1. Gestión Administrativa

\begin{tabular}{|c|c|c|c|c|c|c|}
\hline \multirow{2}{*}{ Ítem } & \multirow{2}{*}{ Opciones } & \multicolumn{5}{|c|}{ Niveles de respuesta } \\
\hline & & 5 & 4 & 3 & 2 & 1 \\
\hline \multirow{2}{*}{$\begin{array}{l}\text { 1. El Aula Invertida, presentó herramientas } \\
\text { para los contenidos y para la comunicación } \\
\text { con calidad técnica en su funcionamiento y } \\
\text { programación. }\end{array}$} & Frec. & 40 & 46 & 10 & 2 & 2 \\
\hline & $\%$ & $40,0 \%$ & $46,0 \%$ & $10,0 \%$ & $2,0 \%$ & $2,0 \%$ \\
\hline 2. El Aula Invertida presentó los & Frec. & 38 & 52 & 6 & 2 & 2 \\
\hline $\begin{array}{l}\text { contenidos en recursos multimedia } \\
\text { (animaciones y actividades, vídeo digital, } \\
\text { videoconferencias, simuladores y libro } \\
\text { electrónico), facilitando esto la comprensión } \\
\text { de los temas. }\end{array}$ & $\%$ & $38,0 \%$ & $52,0 \%$ & $6,0 \%$ & $2,0 \%$ & $2,0 \%$ \\
\hline \multirow{2}{*}{$\begin{array}{l}\text { 3. Los tiempos estipulados en el } \\
\text { cronograma, son los adecuados para dar } \\
\text { cumplimiento al desarrollo de las temáticas. }\end{array}$} & Frec. & 32 & 52 & 10 & 3 & 3 \\
\hline & $\%$ & $32,0 \%$ & $52,0 \%$ & $10,0 \%$ & $3,0 \%$ & $3,0 \%$ \\
\hline \multirow{2}{*}{$\begin{array}{l}\text { 4. El registro e inscripción en elAula Invertida } \\
\text { es sencilla, es de fácil desplazamiento y la } \\
\text { localización de los recursos es evidente. }\end{array}$} & Frec. & 55 & 40 & 1 & 2 & 2 \\
\hline & $\%$ & $55,0 \%$ & $40,0 \%$ & $1,0 \%$ & $2,0 \%$ & $2,0 \%$ \\
\hline
\end{tabular}

Nomenclatura: 5-Totalmente de acuerdo, 4-De acuerdo, 3-Ni de acuerdo, ni en desacuerdo, 2-En desacuerdo, 1-Totalmente en desacuerdo 
La Tabla 1, muestra los ítems del 1 al 4, los cuales hacen referencia a la implementación del aula invertida desde la Gestión Administrativa. Se conoció en el ítem 1, que el $86,0 \%$ de los encuestados están tienen una percepción de aceptación que el aula invertida, presentó herramientas para los contenidos y para la comunicación con calidad técnica en su funcionamiento y programación. En el ítem 2, se comprobó que, el $90,0 \%$ de los estudiantes encuestados, piensan que el aula invertida facilitó la comprensión de los temas, debido que los contenidos se presentaron en recursos multimedia (animaciones y actividades, vídeo digital, videoconferencias, simuladores y libro electrónico). Esta apreciación, se refleja en el cronograma estipulado, debido que en el ítem 3, el $84,0 \%$ de los encuestados afirman estar de acuerdo en que los tiempos son los adecuados para dar cumplimiento al desarrollo de las temáticas. Finalmente, en el ítem 4 se verificó que el 95,0\% de los estudiantes están de acuerdo que el aula invertida es sencilla, además, que es fácil el desplazamiento por ella y la localización de los recursos.

Al observar los resultados, los estudiantes validaron indicadores como calidad de los contenidos, herramientas de comunicación e interacción, cronograma de actividades, y la programación y uso de recursos general. De allí, se infiere que los estudiantes estuvieron satisfechos con el acercamiento y disposición administrativa de los recursos digitales del aula invertida. Lo cual concuerda con el planteamiento de Brunner y Tedesco (2003) quienes sostienen que los procesos de enseñanza y aprendizaje pueden apoyar y acercar las nuevas tecnologías a la escuela, donde el docente desarrolla entornos de aprendizaje logrando utilidad de los recursos tecnológicos para alcanzar los objetivos de aprendizaje.

Tabla 2. Gestión Pedagógica

\begin{tabular}{|c|c|c|c|c|c|c|}
\hline \multirow{2}{*}{ Ítem } & \multirow{2}{*}{ Opciones } & \multicolumn{5}{|c|}{ Opciones de respuesta } \\
\hline & & 5 & 4 & 3 & 2 & 1 \\
\hline \multirow{2}{*}{$\begin{array}{l}\text { 5. La temática planeada en cada una de las } \\
\text { guías fue pertinente, coherente y clara. }\end{array}$} & Frec. & 46 & 42 & 8 & 4 & 0 \\
\hline & $\%$ & $46,0 \%$ & $42,0 \%$ & $8,0 \%$ & $4,0 \%$ & $0,0 \%$ \\
\hline \multirow{2}{*}{$\begin{array}{l}\text { 6. El material interactivo expuesto, es } \\
\text { coherente con cada temática, es claro, con } \\
\text { buena imagen, sonido y de fácil comprensión. }\end{array}$} & Frec. & 32 & 40 & 20 & 4 & 4 \\
\hline & $\%$ & $32,0 \%$ & $40,0 \%$ & $20,0 \%$ & $4,0 \%$ & $4,0 \%$ \\
\hline \multirow{2}{*}{$\begin{array}{l}\text { 7. La metodología didáctica, utilizada en el } \\
\text { Aula Invertida de aprendizaje, atiende a los } \\
\text { distintos estilos de aprendizaje. }\end{array}$} & Frec. & 34 & 46 & 10 & 6 & 4 \\
\hline & $\%$ & $34,0 \%$ & $46,0 \%$ & $10,0 \%$ & $6,0 \%$ & $4,0 \%$ \\
\hline \multirow{2}{*}{$\begin{array}{l}\text { 8. Las animaciones y/o los simuladores del } \\
\text { curso ofrecen retroalimentación y se respeta } \\
\text { la jerarquía de los textos, además de permitir } \\
\text { la impresión de contenidos. }\end{array}$} & Frec. & 17 & 3 & 14 & 28 & 38 \\
\hline & $\%$ & $17,0 \%$ & $3,0 \%$ & $14,0 \%$ & $28,0 \%$ & $38,0 \%$ \\
\hline
\end{tabular}

Nomenclatura: 5-Totalmente de acuerdo, 4-De acuerdo, 3-Ni de acuerdo, ni en desacuerdo, 2-En desacuerdo, 1-Totalmente en desacuerdo 
En la Tabla 2, se da conocer los resultados de los ítems 5 al 8, que da repuesta a la Gestión Pedagógica. Donde se determinó que en el ítem 5 , el $88,0 \%$ de los estudiantes tienen una percepción positiva respecto a que la planeación de la temática junto a las guías didácticas fue pertinente, coherente y clara. Esta apreciación resulta coherente con los resultados del ítem 6 , puesto que el $72,0 \%$ de los encuestados están de acuerdo con que el material interactivo expuesto es claro, puesto que tiene buena resolución de imagen, con adecuado sonido y de fácil comprensión. Asimismo, el 80,0\% manifestaron que la metodología didáctica Aula Invertida, atiende a los distintos estilos de aprendizaje, tal como se menciona en el ítem 7. Se identifica como un aspecto a mejorar, que las animaciones y/o los simuladores del curso ofrecen retroalimentación, así como la jerarquía de los textos y de permitir la impresión de contenidos, es decir, el $66 \%$ de los estudiantes encuestados manifiestan que esta situación no ocurre.

Al examinar estos resultados, se deduce que el aspecto pedagógico, en los ejes metodología y didáctica, los estudiantes consideran estar de acuerdo con la estrategia pedagógica implementada, aunque se debe mejorar en el eje retroalimentación de cada una de las actividades desarrolladas porque las aulas invertidas deben prescindir la instrucción directa de la clase (Johnson \& Renner, 2012), para optimizar los procesos académicos, experiencias de aprendizaje, ocasión para el aprendizaje activo y colaborativo entre los estudiantes.

Tabla 3. Materiales

\begin{tabular}{|c|c|c|c|c|c|c|}
\hline \multirow{2}{*}{ Ítem } & \multirow{2}{*}{ Opciones } & \multicolumn{5}{|c|}{ Opciones de respuesta } \\
\hline & & 5 & 4 & 3 & 2 & 1 \\
\hline \multirow{2}{*}{$\begin{array}{l}\text { 9. Para desarrollar actividades y talleres } \\
\text { de Física prefiere con material multimedia } \\
\text { (videos, web } 2.0 \text { interactiva, libros } \\
\text { electrónicos) }\end{array}$} & Frec. & 12 & 10 & 16 & 36 & 26 \\
\hline & $\%$ & $12,0 \%$ & $10,0 \%$ & $16,0 \%$ & $36,0 \%$ & $26,0 \%$ \\
\hline \multirow{2}{*}{$\begin{array}{l}\text { 10. Para desarrollar actividades y talleres } \\
\text { de Física prefiere con material impreso } \\
\text { (como libros, fotocopias y cuadernos) }\end{array}$} & Frec. & 40 & 38 & 12 & 4 & 6 \\
\hline & $\%$ & $40,0 \%$ & $38,0 \%$ & $12,0 \%$ & $4,0 \%$ & $6,0 \%$ \\
\hline \multirow{2}{*}{$\begin{array}{l}\text { 11. Para desarrollar actividades y } \\
\text { talleres de Física prefiere con sencillos } \\
\text { laboratorios, donde se pueda evidenciar } \\
\text { los fenómenos estudiados. }\end{array}$} & Frec. & 24 & 50 & 14 & 8 & 4 \\
\hline & $\%$ & $24,0 \%$ & $50,0 \%$ & $14,0 \%$ & $8,0 \%$ & $4,0 \%$ \\
\hline
\end{tabular}

Los resultados evidenciados por medio de la Tabla 3 corresponden a la evaluación de los Materiales en el proceso de implementación del Aula Invertida. Se encontró que el $62,0 \%$ de los encuestados rechazaron la utilización del material multimedia en el desarrollo de actividades y talleres de Física (ítem 9), mientras que el porcentaje restante lo ven con cierto grado de favorabilidad. El $78,0 \%$ de los estudiantes prefieren material impreso (como libros, fotocopias y cuadernos) para desarrollar actividades y talleres de Física (ítem 10). Finalmente, el $74,0 \%$ están de acuerdo que al desarrollar actividades y talleres de Física con laboratorios sencillos en donde se pueda 
evidenciar los fenómenos estudiados facilita el entendimiento de los mismos.

Revisando estos resultados, se podría afirmar que el estudio permitió determinar que los materiales utilizados para el estudio de la Física en casa, no eran del agrado de los estudiantes, pareciera que ellos prefieren el acompañamiento permanente del docente para realizarlo (Pozo y Gómez, 2004), ya que la asignatura se podría volver compleja y difícil al estudiarla solos. Por ello, los recursos didácticos suministrados para que el educando aprenda fuera del aula deben extraer datos relevantes, contextualizar los conceptos para desarrollar un entendimiento funcional de los conceptos básicos de la Física (García, Pierce y Quezada, 2008).

Tabla 4. Estilos y habilidades

\begin{tabular}{|l|c|c|c|c|c|c|}
\hline \multirow{2}{*}{ İ́m } & \multirow{2}{*}{ Opciones } & \multicolumn{5}{|c|}{ Opciones de respuesta } \\
\cline { 3 - 7 } & & 5 & 4 & 3 & 2 & 1 \\
\hline $\begin{array}{l}\text { 12. Antes de enfrentarse a una actividad, } \\
\text { reflexiona sobre cómo se resuelve de la } \\
\text { mejor manera. }\end{array}$ & Frec. & 40 & 50 & 10 & 0 & 0 \\
\cline { 2 - 7 } & & $40,0 \%$ & $50,0 \%$ & $10,0 \%$ & $0,0 \%$ & $0,0 \%$ \\
\hline $\begin{array}{l}\text { 13. Sigue los pasos previstos en la } \\
\text { planificación inicial de la tarea. }\end{array}$ & Frec. & 24 & 48 & 24 & 2 & 2 \\
\cline { 2 - 7 } $\begin{array}{l}\text { 14. Evalúa los resultados de su tarea } \\
\text { y reitera la estrategia de resolución de } \\
\text { problemas utilizada inicialmente. }\end{array}$ & Frec. & 20 & 36 & 32 & 12 & 0 \\
\cline { 2 - 7 } $\begin{array}{l}\text { 15. Evalúa los resultados de su tarea e } \\
\text { intenta nuevas vías de solución distintas a } \\
\text { las utilizadas inicialmente. }\end{array}$ & Frec. & 6 & 34 & 24 & 26 & 10 \\
\hline
\end{tabular}

La Tabla 4, permite conocer la percepción de los encuestados respecto a los ítems asociados con la dimensión de Estilos y habilidades. Se resalta que el 90,0\% afirman que antes de realizar la actividad realiza primero una revisión de la mejor forma de resolverlos (ítem 12). Así mismo, el $72,0 \%$ afirman que una vez tienen clara la ruta de solución a la actividad, la ejecutan tal cual como la han diseñado; pero de ellos sólo el $56,0 \%$ realizan retroalimentación de la estrategia seguida una vez obtienen un resultado, y de ese porcentaje sólo el 40,0\% generan una nueva estrategia a partir de los resultados obtenidos.
Los datos obtenidos, dan cuenta que los estudiantes encuestados analizan las actividades, de esta manera realizan una valoración de los resultados obtenidos para buscar nuevas vías de soluciones que impliquen tareas de alto nivel (Talbert, 2014), por ello, los resultados muestran que no todos los educandos siguen pasos, ya que cada estudiante adapta la experiencia de aprendizaje con lo cual la individualiza y diferencia (Davies, Dean y Ball, 2013), centrado en su propio progreso (Davis, 2013). De esta forma, las apreciaciones de los estudiantes en relación evaluar resultados y actividades viene determinada por su propio estilo y técnica de estudio. 
Tabla 5. Interacción en clases sincrónicas y asincrónicas

\begin{tabular}{|c|c|c|c|c|c|c|}
\hline \multirow{2}{*}{ Ítem } & \multirow{2}{*}{ Opciones } & \multicolumn{5}{|c|}{ Opciones de respuesta } \\
\hline & & 5 & 4 & 3 & 2 & 1 \\
\hline \multirow{2}{*}{$\begin{array}{l}\text { 16. Necesita atención altamente } \\
\text { individualizada en el aula, a la hora de } \\
\text { realizar las actividades. }\end{array}$} & Frec. & 20 & 24 & 12 & 26 & 18 \\
\hline & $\%$ & $20,0 \%$ & $24,0 \%$ & $12,0 \%$ & $26,0 \%$ & $18,0 \%$ \\
\hline \multirow{2}{*}{$\begin{array}{l}\text { 17. Necesita del apoyo de otro profesor } \\
\text { u otras herramientas para realizar las } \\
\text { actividades en el aula ordinaria. }\end{array}$} & Frec. & 28 & 42 & 20 & 6 & 4 \\
\hline & $\%$ & $28,0 \%$ & $42,0 \%$ & $20,0 \%$ & $6,0 \%$ & $4,0 \%$ \\
\hline \multirow{2}{*}{$\begin{array}{l}\text { 18. Puede realizar las tareas de forma } \\
\text { autónoma, aunque precise instrucciones } \\
\text { altamente individualizadas por parte del } \\
\text { profesor tutor. }\end{array}$} & Frec. & 46 & 34 & 14 & 2 & 4 \\
\hline & $\%$ & $46,0 \%$ & $34,0 \%$ & $14,0 \%$ & $2,0 \%$ & $4,0 \%$ \\
\hline \multirow{2}{*}{$\begin{array}{l}\text { 19. Pregunta y pide aclaraciones al } \\
\text { maestro en relación con la tarea a realizar. }\end{array}$} & Frec. & 18 & 58 & 8 & 14 & 2 \\
\hline & $\%$ & $18,0 \%$ & $58,0 \%$ & $8,0 \%$ & $14,0 \%$ & $2,0 \%$ \\
\hline \multirow{2}{*}{$\begin{array}{l}\text { 20. Pregunta y pide aclaraciones a sus } \\
\text { compañeros en relación con la tarea a } \\
\text { realizar. }\end{array}$} & Frec. & 4 & 36 & 20 & 10 & 30 \\
\hline & $\%$ & $4,0 \%$ & $36,0 \%$ & $20,0 \%$ & $10,0 \%$ & $30,0 \%$ \\
\hline \multirow{2}{*}{$\begin{array}{l}\text { 21. Prefiere hacer las mismas tareas que } \\
\text { sus compañeros. }\end{array}$} & Frec. & 34 & 10 & 24 & 20 & 6 \\
\hline & $\%$ & $34,0 \%$ & $10,0 \%$ & $24,0 \%$ & $20,0 \%$ & $6,0 \%$ \\
\hline \multirow{2}{*}{ 22. Trabaja mejor cuando está solo. } & Frec. & 20 & 42 & 22 & 10 & 6 \\
\hline & $\%$ & $20,0 \%$ & $42,0 \%$ & $22,0 \%$ & $10,0 \%$ & $6,0 \%$ \\
\hline \multirow{2}{*}{$\begin{array}{l}\text { 23. Trabaja mejor cuando está en pequeño } \\
\text { grupo. }\end{array}$} & Frec. & 30 & 3 & 3 & 54 & 10 \\
\hline & $\%$ & $30,0 \%$ & $3,0 \%$ & $3,0 \%$ & $54,0 \%$ & $10,0 \%$ \\
\hline
\end{tabular}

Nomenclatura: 5-Totalmente de acuerdo, 4-De acuerdo, 3-Ni de acuerdo, ni en desacuerdo, 2-En desacuerdo, 1-Totalmente en desacuerdo

La Tabla 5 permite observar los resultados asociados con la dimensión de Interacción en clases sincrónicas y asincrónicas, dentro del proceso de implementación del Aula Invertida. Se resalta que el $88,0 \%$ de los encuestados manifestaron tener opiniones divididas en cuanto requerir atención y acompañamiento altamente individualizado a la hora realizar las actividades. Se determinó que el $70,0 \%$ afirman requerir apoyo de otro profesor $u$ otras herramientas para realizar las actividades en el aula regular de clases. Asimismo, el $80,0 \%$ de los encuestados dejaron claro que puede realizar tareas de forma autónoma, aunque precise instrucciones altamente individualizadas por parte del profesor tutor; aunque el $76,0 \%$ de ellos prefieren hacer preguntas y pedir aclaraciones al docente con respecto a la actividad a realizar y de ellos sólo el 40,0\% rechazan la opción de pedir ayuda a un compañero de clase en la realización de tareas.

De forma complementaria, se determinó que el $44,0 \%$ prefieren hacer las mismas tareas que sus compañeros. Un hallazgo se dio en el hecho de que el $62,0 \%$ aseguran que trabajan mejor cuando están solos, en contraste con el $33,0 \%$ quienes afirman que les gusta trabajar en pequeños grupos.

Estos resultados permiten resaltar que los estudiantes encuestados precisan orientaciones por el profesor (cara a cara) para aclarar dudas en el desarrollo de actividades escolares, lo que pudiera ser una falencia que se denota en la organización y estructuración de tareas junto a 
los contenidos e instrucción en línea, por lo que se hace necesario la aplicación de métodos que les ayuden a ser eficientes y efectivos en sus labores académicas. No obstante, los resultados encontrados en este estudio confirman que la estrategia de invertir el aprendizaje es una buena opción que ayuda en el desarrollo de habilidades y competencias de cualquier área, aunque se debe culturizar al estudiante en el uso de medios audiovisuales para aprender; en el cual incluyen estrategias colaborativas y aprendizaje activo, en la resolución de problemas (Berrett, 2012).

\section{CONCLUSIONES}

Terminado el proceso de implementación del método de Aula Invertida, como estrategia para apoyar la asignatura de Física se podría concluir que aproximadamente el $86,0 \%$ de los estudiantes se manifestaron satisfechos con el desarrollo de la gestión administrativa, permitiendo evidenciar que se necesita de los docentes la implementación de mecanismos de comunicación e interacción a través de plataformas, redes, blogs, entre otras; y con estos recursos poder ampliar los canales para la orientación de las temáticas.

Encuantoal rendimientoacadémico, comparando los periodos I III, hubo un mejoramiento promedio del $15,0 \%$ y $17,0 \% \%$ para los grados Décimo y Undécimo respectivamente, lo cual trajo como producto una reducción del nivel de reprobados. En los grupos analizados, se encontró que un $17,0 \%$ de estudiantes se sienten molestos con este tipo de metodologías y tareas fuera del aula, por lo que el docente debe motivar a aquellos que se muestran apáticos a acceder a dichos entornos tecnológicos de aprendizaje en dónde interactúan con los diversos recursos tecnológicos.

Otro aspecto relevante es el material con que el estudiante prefiere aprender, ya que el $62,0 \%$ de los estudiantes manifestaron que prefieren conceptualizar y documentarse con elementos diferentes a los recursos multimedia, videos y libros electrónicos; por lo que el docente debe organizar los ejes temáticos incorporando este tipo de recursos que resultan valiosos cuando se limita el trabajo presencial o sincrónico en el aula. La estrategia aplicada de invertir el aula de clase es un modelo mediado por la tecnología que es productiva e innovadora, ya que permite la combinación de materiales, metodologías y estrategias que apoya el desarrollo de aprendizaje en cualquier área del conocimiento.

\section{REFERENCIAS BIBLIOGRÁFICAS}

Bergmann, J. \& Sams, A., (2012). Flip Your Classroom. Reach Every Student in Every Class Every Day. Washington, USA: ISTEASCD.

Berrett, D. (2012). How 'flipping' the classroom can improve the traditional lecture. The chronicle of higher education. http://www. chronicle.com/article/How-Flipping-theClassroom/130857/

Bloom, B. (1964). Características humanas y aprendizaje escolar. Bogotá: Voluntad

Christensen, C. M., Horn, M. B., \& Staker, H. (2013). Is K-12 Blended Learning Disruptive? An Introduction to the Theory of Hybrids. (Clayton Christensen Institute for Disruptive Innovation)

Davis, C. (2013). Flipped or inverted learning: Strategies for course design. In E. G. Smyth \& J. X. Volker (Eds.), Enhancing instruction with visual media: Utilizing video and lecture capture (pp. 241-265). Hershey, PA: IGI Global.

Davies, R. S., Dean, D. L. \& Ball, N. (2013). Flipping the classroom and instructional technology integration in a college-level information systems spreadsheet course. Education Tech Research Dev, 61, 563-580. https://doi.org/10.1007/s11423-013-9305-6 
Durley, C., (2014). Flipped Classroom Transitions to Deep Learning, in Flipped learning: Gateway to student engagement by J. Bergmann y A. Sams, International Society for Technology in Education, pp. 1-182, Oregon, USA.

Echazarra, A. y Schwabe, M. (2019). Colombia - Country Note - PISA 2018 Results. París: OECD Publishing. https://www.oecd.org/ pisa/publications/PISA2018_CN_COL_ESP. pdf

Flores-García, S., Chávez-Pierce, J., LunaGonzález, J., González-Quezada, M., González-Demoss, M., \& HernándezPalacios, A. (2015). El aprendizaje de la física y las matemáticas en contexto. Cultura Científica y Tecnológica, O(24).

Gamboa-Suárez, A., Hernández-Suárez, C., y Prada-Núñez, R. (2018). Práctica pedagógica y competencias TIC. Saber, Ciencia y Libertad, 13(1), 258-274. https://doi.org/10.18041/2382-3240/ saber.2018v13n1.2090

Gaviria, D., Arango, J., Valencia, A. y Bran, L. (2019). Percepción de la Estrategia Aula Invertida en Escenarios Universitarios. Revista mexicana de investigación educativa, 24(81), 593-614.

Hernández, C. A., Prada, R., \& Ramírez, P. (2018). Perspectivas actuales de los docentes de Educación Básica y Media acerca de la aplicación de las Competencias Tecnológicas en el aula. Revista Espacios, 39(43), 19.

Hernández-Suárez, C. A., Prada-Núñez, R. y Gamboa-Suárez, A. (2020). Formación inicial de maestros: Escenarios activos desde una perspectiva del aula invertida. Formación universitaria, 13(5), 213-222. https://dx.doi. org/10.4067/S0718-50062020000500213
Johnson, L. W., \& Renner, J. D. (2012). Effect of the flipped classroom model on a secondary computer applications course: Student and teacher perceptions, questions and student achievement (University of Louisville).

Kolb, D. A. (1984). Experiential learning: experience as the source of learning and development. Englewood Cliffs, NJ: Prentice Hall.

Lage, M. J., Platt, G. J., \& Treglia, M. (2000). Inverting the classroom: A gateway to creating an inclusive learning environment. The Journal of Economic Education, 31, 3043.

Martín, M. M., Hernández-Suarez, C. A., y Mendoza-Lizcano, S. M. (2017). Ambientes de aprendizaje basados en herramientas web para el desarrollo de competencias TIC en la docencia. Revista Perspectivas, 2(1), 97-104. https://doi.org/10.22463/25909215.1282

Martínez-Olvera, W., Esquivel-Gámez, I., y Martínez, J. (2015a). Aula invertida o modelo invertido de aprendizaje: origen, sustento e implicaciones. En I., Esquivel-Gámez (Coord.), Los modelos Tecno-Educativos, revolucionando el aprendizaje del siglo XXI (pp. 137-154).

Martínez-Olvera, W., Esquivel-Gámez, I., y Martínez, J. (2015b). Acercamiento teóricopráctico al modelo de aprendizaje invertido. En M. Osorio \& I. E. Peón (Eds.), Alternativas para nuevas prácticas educativas (pp. 158172). México: Amapsi Editorial.

Mora-Ramírez, $\quad$ B., \& Hernández-Suárez, C. (2017). Las aulas invertidas: una estrategia para enseñar y otra forma de aprender física. INVENTUM, 12(22), 4251. https://doi.org/10.26620/uniminuto. inventum.12.22.2017.42-51 
Piaget, J. (1972). The Psychology of Intelligence. Totowa, NJ: Littlefield.

Pozo J. I. y Gómez M. (2004). Aprender y enseñar ciencia: del conocimiento cotidiano al conocimiento científico. España: Morata.

Prada, R., Hernández, C. A., \& Gamboa, A. A. (2019, November). Different scenarios for the teaching of mathematics with the support of virtual platforms: Flipped classroom. In Journal of Physics: Conference Series (Vol. 1388, No. 1, p. 012046). IOP Publishing.

Talbert, R. (2014). Inverting the linear algebra classroom. Primus: Problems, Resources, and Issues in Mathematics Undergraduate Studies, 24(5), 361-374. http://www. tandfonline.com/doi/pdf/10.1080/10511970.2 $\underline{014.883457}$

Vigostky, L. (1978). Pensamiento y lenguaje. La Habana: Editorial Revolucionaria.

Zainuddin, Z. y Halili, S. (2016). Flipped Classroom Research and Trends from Different Fields of Study. The International Review of Research in Open and Distributed Learning, 17(3), 313-340. https://doi. org/10.19173/irrodl.v17i3.2274 